Copyright(C 2005 IEEE. Reprinted from Proceedings of IEEE Workshop on Applications of Signal Processing to Audio and Acoustics, pp.191-194, New Paltz, NY, USA, October 2005.

This material is posted here with permission of the IEEE. Such permission of the IEEE does not in any way imply IEEE endorsement of your university's web sites products or services. Internal or personal use of this material is permitted. However, permission to reprint/republish this material for advertising or promotional purposes or for creating new collective works for resale or redistribution must be obtained from the IEEE by writing to pubs-permissions@ieee.org.

By choosing to view this document, you agree to all provisions of the copyright laws protecting it. 


\title{
IMPROVED ADJUSTABLE BOUNDARY CONDITION FOR THE 3-D DIGITAL WAVEGUIDE MESH
}

\author{
Antti Kelloniemi* \\ Telecommunications Software and Multimedia Laboratory \\ Helsinki University of Technology \\ P.O. Box 5400, 02015 TKK, Finland \\ antti.kelloniemi@tkk.fi
}

\begin{abstract}
The digital waveguide (DWG) mesh is a method for simulating wave propagation in multiple dimensions. In threedimensional form it can be used for modeling room acoustics or resonating bodies of musical instruments, for example. Until now boundary conditions in the threedimensional DWG mesh have been implemented using methods originating from the updating functions of a onedimensional digital waveguide. A new boundary structure, which better takes into account the 3-D mesh topology, is now introduced. With this new method, the reflection magnitude is shown to match the desired value over a much wider range of reflection coefficient values than with the earlier method.
\end{abstract}

\section{INTRODUCTION}

For optimal results, the method for modeling sound propagation has to be chosen taking into account the size of the object, frequency bandwidth, amount of detail needed, and many other model parameters. In room acoustics modeling, geometrical methods such as ray tracing and image source methods are often used for modeling the first reflections. The late reverberation is then modeled using statistical methods or delay networks, for example.

Unlike these methods, mesh-based algorithms such as the digital waveguide (DWG) mesh can be used for simulating the whole impulse response. Because the mesh is a strict physical model of the acoustic space, the timing and arrival directions of the direct and reflected signals are modeled correctly. The recursive nature of the mesh structure enables modeling of a high number of reflections without a growing demand for memory. As the mesh algorithm inherently models diffraction and interference effects, it is suitable for modeling sound propagation also in low frequencies, where geometrical methods typically fail.

* The author has received funding from the Academy of Finland (project no. 201050) and the Nokia Foundation. He would like to extend his thanks to Patty Huang and Vesa Välimäki for their valuable comments.
The mesh topology can be chosen in many ways. The interpolated rectilinear topology [1] discussed in Section 2 is used in this work. The Cartesian grid makes it relatively easy to handle and the interpolation scheme diminishes remarkably the direction dependent dispersion in the mesh.

Currently an exact solution for implementing boundaries in a multi-dimensional DWG mesh does not exist. The 1-D boundary implementations [2] can be used as discussed in Section 3.1, but the change in dimensionality results in large errors. Better approximations have been suggested for the 2-D mesh $[3,4,5,6]$, but these have not been extended to three dimensions until now.

The new adjustable boundary condition introduced in Section 3.2 is based on the admittance boundary method introduced previously for a two-dimensional mesh [6]. The boundary is modeled by weighting the signal values with admittance coefficients at the boundary location. Part of the signal is reflected at the discontinuity while the other part passes through. Behind the admittance boundary, the mesh is truncated with an absorbing boundary condition. The performance of the new method is tested in simulations described in Section 4.

\section{INTERPOLATED 3-D DIGITAL WAVEGUIDE MESH}

The digital waveguide mesh discussed in this paper is a computational method for approximating multidimensional wave propagation $[7,8,2]$. A one-dimensional digital waveguide is constructed by connecting two parallel, bi-directional delay lines together with scattering junctions. It can be used for modeling the wave propagation in, for example, strings and acoustical tubes.

This structure is extended to multiple dimensions by joining more delay lines together. A DWG mesh is constructed having a scattering junction at each node location along a regular grid, separated with bi-directional unit delays from its neighboring junctions. This makes it possible to model wave propagation in 2-D objects such as mem- 
branes and plates or 3-D structures such as bodies of musical instruments or rooms.

Sound propagation is simulated by updating signal values in the mesh at each time step. The updating functions were first derived for the traveling wave formulation (Wmesh), where two values of variables representing the wave decomposition of the signal are saved at each interconnection and physical signal values at the node points are computed as a weighted sum of those [8].

Later the Kirchhoff variable formulation (K-mesh) was derived, where the physical signal values are used as such [2]. It has been shown to be functionally equivalent with the W-mesh [9]. In a non-interpolated rectilinear 3-D Kmesh, the value at a node location at current time step $n$ is computed as a weighted sum of the values of its six axial neighbors at previous time step $n-1$ and its own value at time step $n-2$. As only two values per node location are saved, considerable memory savings are obtained when compared to the $\mathrm{W}$-mesh.

For a 3-D K-mesh junction, the updating function is written as

$$
p_{\mathrm{c}}(n)=\frac{\sum_{l=1}^{6} Y_{l} p_{l}(n-1)}{\frac{1}{2} \sum_{l=1}^{6} Y_{l}}-p_{\mathrm{c}}(n-2),
$$

where $Y$ is the characteristic admittance of an interconnection, $p$ is the signal value at a junction, subscript $\mathrm{c}$ denotes the junction to be calculated and index $l$ denotes its axial neighbors. The maximum simulation frequency $f$ is restricted to a quarter of the sampling frequency $f_{\mathrm{s}}$ of the mesh.

In the non-interpolated mesh, wave propagation is limited to axial directions. Dispersion error is caused by nonuniform distances from neighboring nodes to the center node. To minimize this error, the center node value and the values of its 20 diagonal neighbors at time step $n-1$ are used to interpolate the signal values at unit delay distances from the center node [1]. Combining these to (1) results in

$$
\begin{aligned}
p_{\mathrm{c}}(n)= & \frac{\sum_{k=1}^{3} \sum_{l=1}^{3} \sum_{m=1}^{3} Y_{k, l, m} h_{k, l, m} p_{k, l, m}(n-1)}{\frac{1}{2} \sum_{k=1}^{3} \sum_{l=1}^{3} \sum_{m=1}^{3} Y_{k, l, m} h_{k, l, m}} \\
& -p_{\mathrm{c}}(n-2),
\end{aligned}
$$

where $h_{k, l, m}$ are the interpolation coefficients for each node value and indexes $k, l$ and $m$ run through all neighboring nodes in $\mathrm{x}, \mathrm{y}$ and $\mathrm{z}$ directions, respectively, as well as the center node. In homogeneous media the admittances of all interconnections are equal, so (2) reduces to

$p_{\mathrm{c}}(n)=\frac{2}{26} \sum_{k=1}^{3} \sum_{l=1}^{3} \sum_{m=1}^{3} h_{k, l, m} p_{k, l, m}(n-1)-p_{\mathrm{c}}(n-2)$.

For determining the interpolation coefficient values, linear interpolation based on the distances from the center node to its neighboring nodes is used. The weighting coefficients are $h_{a}=2 \sqrt{2}-\frac{1}{2}$ for the 6 axial neighbors, $h_{2 d}=\frac{3}{4}$ for the 12 2-D diagonal neighbors and $h_{3 d}=\frac{1}{8}$ for the 8 3-D diagonal neighbors furthest away from the center node [10]. The weighting coefficient value of the center node depends on the admittance coefficient values of the diagonal connections, resulting in

$$
h_{\mathrm{c}}=\left(\frac{3}{2}-\sqrt{2}\right) \sum^{12} Y_{2 \mathrm{~d}}+\frac{1}{8} \sum^{8} Y_{3 \mathrm{~d}},
$$

where $Y_{2 \mathrm{~d}}$ and $Y_{3 \mathrm{~d}}$ are the admittances of each of the 2-D and 3-D diagonal connections, respectively.

\section{BOUNDARY CONDITIONS}

A method for determining the reflection coefficient at a discontinuity of wave propagation media is needed for modeling spaces with structures such as walls, air ducts or furniture. Methods for controllable termination of the mesh are of special interest as computational effort is saved by truncating the mesh to match the size of the simulation space.

A new method is introduced for this use, where the reflection coefficient at the boundary can be set to a constant real value $r$ for each interconnection between the boundary and its neighboring nodes.

\subsection{Previous method: 1-D boundary}

The boundary condition in a DWG mesh is constructed by adding special boundary nodes outside the mesh edges to provide the desired reflection characteristics. Originally the boundary nodes have been connected to only one neighboring node at the edge of the mesh. Irrespective of the model dimensionality or complexity of the topology, their values have been computed by the one-dimensional rule

$$
p_{\mathrm{B}}(n)=(1+r) p_{1}(n-1)-r p_{\mathrm{B}}(n-2),
$$

where subscript B denotes the boundary node and subscript 1 represents its perpendicular neighbor [2]. The two terms on the right hand side of (5) represent the signal value at the mesh edge one time step ago and the signal value reflected from the boundary node two time steps ago, respectively.

It has been noted that in a multi-dimensional mesh this 1-D boundary structure gives good results only with high absolute values of the reflection coefficient [5]. Results with $r$ values close to zero are poor due to the reflection caused by the change in mesh topology at the boundary.

The performance at low reflection coefficient values was improved later by more complex boundary node updating functions [3, 4]. Unfortunately these have been optimized only for the 2-D mesh and cause unstability in the interpolated 3-D mesh. Until now the best known method for terminating a 3-D mesh has been the one-dimensional boundary condition defined by (5). 


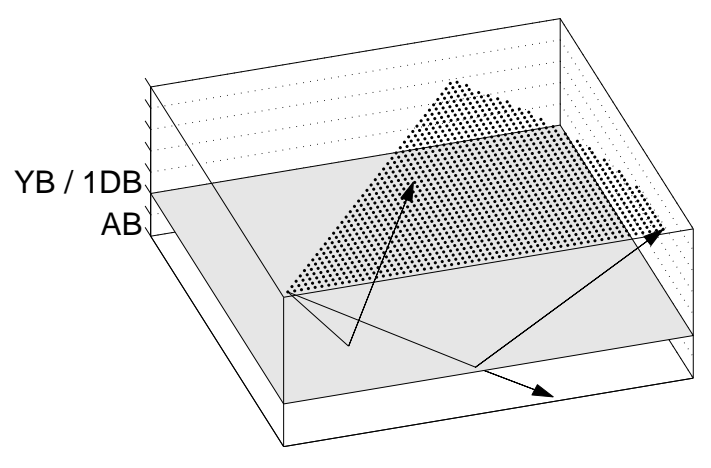

Figure 1: A portion of the simulated mesh: The signal reflected from the admittance boundary $(Y B)$ or the $1-D$ boundary $(1 D B)$ was received at 1273 nodes indicated with dots. For the admittance boundary simulation, the mesh was truncated with an absorbing boundary $(A B)$.

\subsection{New method: Admittance boundary}

As discussed in Section 2, changes in the wave propagation media can be modeled using the characteristic admittance constants in the calculation of a junction value. Earlier this method was utilized only in the interior of a mesh. As part of the signal gets passed through the admittance discontinuity, this method alone does not truncate the mesh. On the other hand, absorbing boundaries have been implemented for modeling openings at the mesh edges. No reflections are wanted when waves propagate out of the simulation space.

Similarly as earlier proposed for a 2-D mesh [6], a new improved boundary structure is obtained by combining these two methods - an admittance change for the reflective boundary and an absorbing boundary behind it to truncate the mesh.

In the updating function of the admittance boundary junctions, the values of the neighboring junctions in the direction of the mesh edge are multiplied with the admittance value

$$
Y=\frac{1-r}{1+r},
$$

where $r$ is the desired reflection coefficient [11]. At the limit, where $r=-1, Y$ goes to infinity. In this case a large constant value is used instead. The center node weight is defined accordingly by (4). No extra delays are implemented at the admittance boundary, so no error is introduced to the signal phase or to the mesh size.

The signal propagated through the admittance boundary is absorbed at the mesh edge using the absorbing boundary condition derived from (5) with $r=0$,

$$
p_{\mathrm{B}}(n)=p_{1}(n-1) .
$$

For stability, at least one node layer has to be left between the admittance boundary and absorbing boundary so that the admittance change location is out of the reach of the absorbing boundary update function (7).

This new boundary structure also allows direction dependence to be implemented, by defining individual constants for each interconnection. Since material parameters are often given as constants in octave bands, simulations could be run separately for each of them for modeling frequency-dependent responses. Some error is expected to arise due to the signal reflected back from the non-optimal absorbing boundary. Still, at positive values of $r$ the error is expected to be minor, since the input to the admittance boundary junctions from the direction of the absorbing boundary is weighted with small values of $Y$.

\section{COMPARISON SETUP AND RESULTS}

The two methods were compared by simulations using an interpolated 3 -D mesh of $45 \times 75 \times 110$ junctions. The simulation was repeated for 101 reflection coefficient values, at $r=-1 \ldots 1$. Each simulation was run for 100 time steps.

The mesh was initialized at the point $(7,35,35), 5$ nodes away from the admittance boundary, with a unit impulse filtered with transfer function $1-z^{2}$ as earlier shown optimal [9]. The test signal at incident angles $0^{\circ} \ldots 80^{\circ}$ was received at 1273 points located at the same distance from the tested boundary as indicated in Fig. 1. Receiving points at incident angles significantly over $80^{\circ}$ would require large mesh sizes, so those were omitted. Due to the symmetry of the mesh topology, node values only within $1 / 8$ of the full circle were needed to resolve the reflection magnitude to all rotation directions.

The level of the signal radiated directly from the source to the receivers was subtracted from the data. The last half of the received signal was windowed with the right half of the Hanning function to avoid truncation error in calculation of the spectra. For resolving the achieved reflection coefficient value, the resulting signal level was compared to the level of a signal representing ideal and lossless reflection. This reference signal was obtained at a mirror image location on the other side of the admittance boundary when the boundary was set to be non-reflective $(Y=1)$ and the mesh was made large enough to avoid reflections from the edges.

The maximum error at incident angles from $0^{\circ}$ to $80^{\circ}$ plotted in Fig. 2 was found for each frequency value from all the computed magnitude responses. The absolute error of the achieved reflection magnitude was calculated by subtracting the set value of $r$ from the frequency responses. The relative error was calculated by dividing the absolute error value by $r$.

The new method is seen to outperform the old method. The difference is most significant with the positive values of $r$, where the absolute error value is less than 0.1 within the frequency band $0.01<f<0.18$ at $r>0.3$ with the 

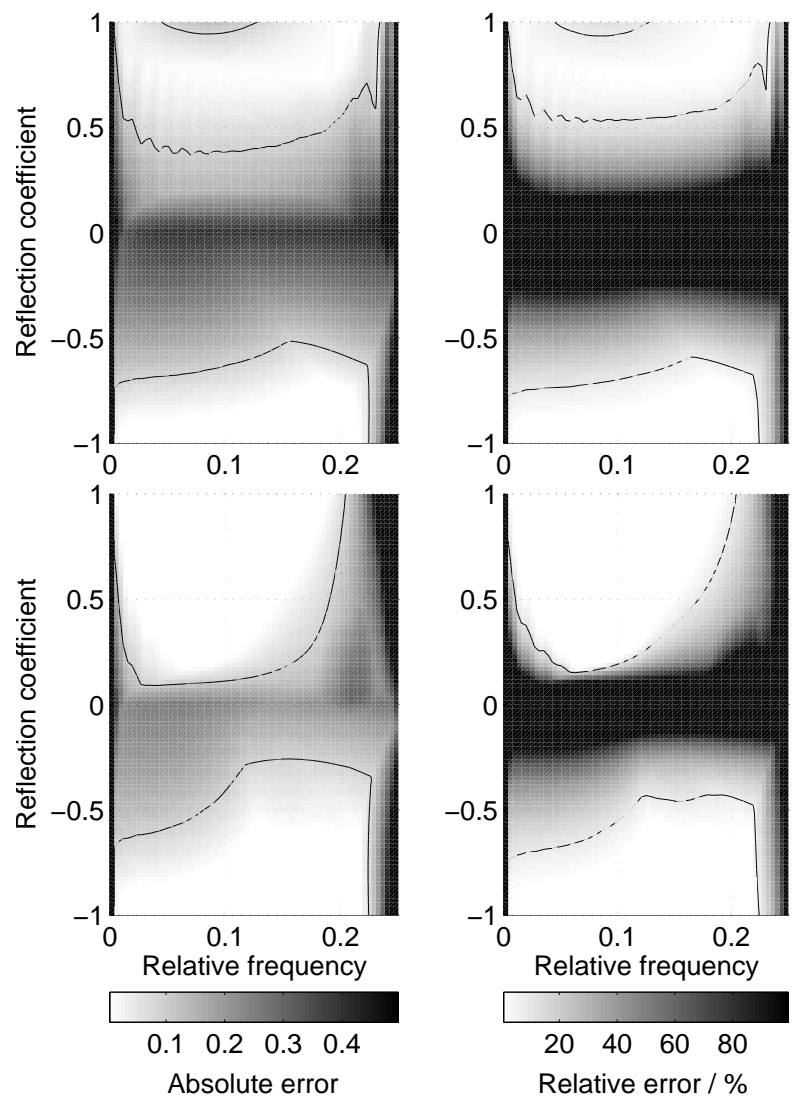

Figure 2: Maximum absolute and relative error of the reflection coefficient value in the original adjustable boundary condition (top) and in the new boundary condition (bottom) at incident angles $0^{\circ} \leq \theta<80^{\circ}$. Contour lines are drawn at absolute error value 0.1 and relative error value $10 \%$.

new method. The old method performs equally well only at $0.5<r<0.95$. With negative values of $r$, the error remains below 0.1 at frequencies $0.01<f<0.23$ at $r<-0.6$ with the new method but at $r<-0.7$ with the old method. At lower absolute values of $r$, the accuracy is acceptable over a narrower frequency band, but still the new method gives significantly better results when compared to the old method.

\section{CONCLUSIONS}

A new boundary structure has been introduced for the interpolated 3-D DWG mesh. It was shown in simulation results that with the new method the reflection coefficient can be realized much more accurately than with a previous method. The structure also offers the possibility to define direction-dependent reflection coefficients. The performance of this method would benefit further from a better absorbing boundary, which will be pursued in future research.

\section{REFERENCES}

[1] L. Savioja and V. Välimäki, "Interpolated rectangular 3-D digital waveguide mesh algorithms with frequency warping," IEEE Trans. Speech and Audio Processing, vol. 11, no. 6, pp. 783-790, November 2003.

[2] L. Savioja, T. Rinne, and T. Takala, "Simulation of room acoustics with a 3-D finite difference mesh," in Proc. Int. Computer Music Conf. (ICMC), Aarhus, Denmark, September 1994, pp. 463-466.

[3] D. T. Murphy and J. Mullen, "Digital waveguide mesh modelling of room acoustics: improved anechoic boundaries," in Proc. Int. Conf. Digital Audio Effects (DAFx), Hamburg, Germany, September 2002, pp. $163-168$.

[4] A. Kelloniemi, D. T. Murphy, L. Savioja, and V. Välimäki, "Boundary conditions in a multidimensional digital waveguide mesh," in Proc. IEEE Int. Conf. Acoustics, Speech, and Signal Processing (ICASSP), vol. 4, Montreal, Canada, May 2004, pp. $25-28$.

[5] A. Kelloniemi, L. Savioja, and V. Välimäki, "Spatial filter-based absorbing boundary for the 2-D digital waveguide mesh," IEEE Signal Processing Letters, vol. 12, no. 2, pp. 126-129, February 2005.

[6] A. Kelloniemi, "Improved adjustable boundary condition for the 2-D digital waveguide mesh," in Proc. Int. Conf. Digital Audio Effects (DAFx), Madrid, Spain, September 2005.

[7] J. O. Smith, "Physical modeling using digital waveguides," Computer Music Journal, vol. 16, no. 4, pp. 74-91, 1992.

[8] S. Van Duyne and J. O. Smith, "Physical modeling with the 2-D digital waveguide mesh," in Proc. Int. Computer Music Conf. (ICMC), Tokyo, Japan, September 1993, pp. 40-47.

[9] M. Karjalainen and C. Erkut, "Digital waveguides vs. finite difference schemes: Equivalence and mixed modeling," EURASIP Journal on Applied Signal Processing, no. 7, pp. 978-989, June 2004.

[10] L. Savioja, "Improving the three-dimensional digital waveguide mesh by interpolation," in Proc. Nordic Acoustical Meeting (NAM), Stockholm, Sweden, September 1998, pp. 265-268.

[11] H. Kuttruff, Room acoustics, 4th ed. London: Spon Press, 2000. 